\title{
Remarks on Caristi's fixed point theorem in metric spaces with a graph
}

\author{
Monther Rashed Alfuraidan*
}

Dedicated to Munairh Ali Alsaqer.

"Correspondence:

monther@kfupm.edu.sa

Department of Mathematics and

Statistics, King Fahd University of

Petroleum and Minerals, Dhahran,

31261, Saudi Arabia

\begin{abstract}
We discuss some extensions of Caristi's fixed point theorem for mappings defined on a metric space endowed with a graph. This work should be seen as a generalization of the classical Caristi's fixed point theorem where the assumptions in Caristi's theorem can, a priori, be weakened. It extends some recent works on Caristi's fixed point theorem for mappings defined on metric spaces with a graph.

MSC: Primary 47H09; secondary 46B20; 47H10; 47E10

Keywords: acyclic digraph; Banach contraction principle; Caristi; directed graph; fixed point; partially ordered metric spaces
\end{abstract}

\section{Introduction}

This work was motivated by some recent work on Caristi's fixed point theorem for mappings defined on metric spaces with a graph [1]. It seems that the terminology of graph theory instead of partial ordering gives clearer pictures and yields generalized fixed point theorems. This paper is kind of a revisit of [2] in graph theory terminology.

Caristi's fixed point theorem may be one of the most beautiful extensions of the Banach contraction principle [3, 4]. Recall that this theorem states that any map $T: M \rightarrow M$ has a fixed point provided that $M$ is a complete metric space, and there exists a lower semicontinuous map $\phi$ mapping $M$ into the nonnegative numbers such that

$$
d(x, T x) \leq \phi(x)-\phi(T x)
$$

for every $x \in M$. Recall that $x \in M$ is called a fixed point of $T$ if $T(x)=x$. This general fixed point theorem has found many applications in nonlinear analysis. It is shown, for example, that this theorem yields essentially all the known inwardness results [5] of geometric fixed point theory in Banach spaces. Recall that inwardness conditions are the ones which assert that, in some sense, points from the domain are mapped toward the domain. Possibly the weakest of the inwardness conditions, the Leray-Schauder boundary condition, is the assumption that a map points $x$ of $\partial M$ anywhere except to the outward part of the ray originating at some interior point of $M$ and passing through $x$.

The proofs given to Caristi's result vary and use different techniques (see [3, 6-8]). It is worth to mention that because of Caristi's theorem close connection to Ekeland's [9] variational principle, many authors refer to it as the Caristi-Ekeland fixed point theorem.

○2014 Alfuraidan; licensee Springer. This is an Open Access article distributed under the terms of the Creative Commons Attribution License (http://creativecommons.org/licenses/by/2.0), which permits unrestricted use, distribution, and reproduction in any medium, provided the original work is properly cited. 
For more on Ekeland's variational principle and the equivalence between Caristi-Ekeland fixed point result and the completeness of metric spaces, the reader is advised to read [10]. In this work we present a characterization to the existence of minimal elements in partially ordered sets in terms of fixed point of multivalued maps, see [2]. In fact, we state that the assumptions on the graph $G$ needed to insure the existence of the minimal element among its vertices. Then we show how Caristi's theorem in metric spaces with a graph may be generalized.

\section{Minimal points and fixed point property in a graph}

Let $A$ be an abstract set partially ordered by $\prec$. We will say that $a \in A$ is a minimal element of $A$ if and only if $b \prec a$ implies $b=a$. The concept of minimal element is crucial in the proofs given for Caristi's fixed point theorem.

Theorem 2.1 [2] Let $(A, \prec)$ be a partially ordered set. Then the following statements are equivalent.

(1) A contains a minimal element.

(2) Any multivalued map $T$ defined on $A$, such that for any $x \in A$ there exists $y \in T x$ with $y \prec x$, has a fixed point, i.e., there exists a in $A$ such that $a \in T(a)$.

Remark 2.1 Recall that Taskovic [11] showed that Zorn's lemma is equivalent to the following:

(TT) Let $\mathcal{F}$ be a family of self-mappings defined on a partially ordered set $A$ such that

$$
x \leq f(x) \quad(\text { resp. } f(x) \leq x)
$$

for all $x \in A$ and all $f \in \mathcal{F}$. If each chain in $A$ has an upper bound (resp. lower bound), then the family $\mathcal{F}$ has a common fixed point.

Therefore, Theorem 2.1 is different from the remark as theorem [2] considers the existence of minimal elements, which in general does not imply that any chain has a lower bound.

Throughout this section we assume that $(X, d)$ is a metric space, and $G$ is a directed graph (digraph) with a set of vertices $V(G) \subseteq X$ and a set of edges $E(G)$ contains all the loops, i.e., $(x, x) \in E(G)$ for any $x \in V(G)$. We also assume that $G$ has no parallel edges (arcs) and so we can identify $G$ with the pair $(V(G), E(G))$. Our graph theory notations and terminology are standard and can be found in all graph theory books, like $[12,13]$. A digraph $G$ is called an oriented graph if whenever $(u, v) \in E(G)$, then $(v, u) \notin E(G)$.

Let $(X, \preceq)$ be a partially ordered set. We define the oriented graph $G_{\preceq}$ in $X$ as follows: the vertices of $G_{\leq}$are the elements of $X$, and two vertices $x, y \in X$ are connected by a directed edge ( $\operatorname{arc})$ if $x \leq y$. Therefore, $G_{\leq}$has no parallel arcs as $x \leq y$ and $y \preceq x \Rightarrow x=y$.

If $x, y$ are vertices of the digraph $G$, then a directed path from $x$ to $y$ of length $N$ is a sequence $\left\{x_{i}\right\}_{i=0}^{N}$ of $N+1$ vertices such that

$$
x_{0}=x, \quad x_{N}=y \quad \text { and } \quad\left(x_{i}, x_{i+1}\right) \in E(G), \quad i=0,1, \ldots, N .
$$

A closed directed path of length $N>1$ from $x$ to $y$, i.e., $x=y$, is called a directed cycle. An acyclic digraph is a digraph that has no directed cycle. 
Given an acyclic digraph $G$, we can always define a partial order $\preceq_{G}$ on the set of vertices of $G$ by $x \preceq_{G} y$ whenever there is a directed path from $x$ to $y$.

In the next result we discuss a common fixed point theorem with a graph. Let $\phi: X \rightarrow$ $[0, \infty)$ be a map and define the order $\prec_{\phi}$ (see $\left.[3,14,15]\right)$ on $X$ by

$$
x \prec_{\phi} y \quad \text { iff } \quad d(x, y) \leq \phi(y)-\phi(x)
$$

for any $x, y$ in $X$. It is straightforward to see that $\left(X, \prec_{\phi}\right)$ is a partially ordered set. However, it is not clear what are the minimal assumptions in $X$ and $\phi$ which ensures the existence of a minimal element. In particular, if $X$ is complete and $\phi$ is lower semi-continuous, then any decreasing chain in $\left(X, \prec_{\phi}\right)$ has a lower bound. Indeed, let $\left(x_{\alpha}\right)_{\alpha \in \Gamma}$, where $\Gamma$ is the set of all ordinals, be a decreasing chain, then $\left(\phi\left(x_{\alpha}\right)\right)_{\alpha \in \Gamma}$ is a decreasing net of positive numbers. Let $\alpha_{n}$ be an increasing sequence of elements from $\Gamma$ such that

$$
\lim _{n \rightarrow \infty} \phi\left(x_{\alpha_{n}}\right)=\inf \left\{\phi\left(x_{\alpha}\right): \alpha \in \Gamma\right\} .
$$

Using the definition of $\prec_{\phi}$, one can easily show that $x_{\alpha_{n}}$ is a Cauchy sequence and therefore converges to $x \in X$. Finally, it is straightforward to see that $x \prec_{\phi} x_{\alpha_{n}}$ for all $n \geq 1$, which means that $x$ is a lower bound for $\left(x_{\alpha_{n}}\right)_{n \geq 1}$. In order to see that $x$ is also a lower bound for $\left(x_{\alpha}\right)_{\alpha \in \Gamma}$, let $\beta \in \Gamma$ be such that $x_{\beta} \prec_{\phi} x_{\alpha_{n}}$ for all $n \geq 1$. Then we have $\phi\left(x_{\beta}\right) \leq \phi\left(x_{\alpha_{n}}\right)$ for all $n \geq 1$, which implies $\phi\left(x_{\beta}\right)=\inf \left\{\phi\left(x_{\alpha}\right): \alpha \in \Gamma\right\}$. Since $d\left(x_{\beta}, x_{\alpha_{n}}\right) \leq \phi\left(x_{\alpha_{n}}\right)-\phi\left(x_{\beta}\right)$, we get $\lim _{n \rightarrow \infty} x_{\alpha_{n}}=x_{\beta}$, which implies $x_{\beta}=x$. Therefore, for any $\alpha \in \Gamma$, there exists $n \geq 1$ such that $x_{\alpha_{n}} \prec_{\phi} x_{\alpha}$, which implies $x \prec_{\phi} x_{\alpha}$, i.e., $x$ is a lower bound of $\left(x_{\alpha}\right)_{\alpha \in \Gamma}$. Zorn's lemma will therefore imply that $\left(X, \prec_{\alpha}\right)$ has minimal elements.

Corollary 2.1 [2] Let $(X, d)$ be a metric space and $\phi: X \rightarrow[0, \infty)$ be a map. Consider the partially ordered set $\left(X, \prec_{\phi}\right)$. Assume that $a \in X$ is a minimal element. Then any map $T: X \rightarrow X$ such that for all $x \in X$

$$
d(x, T x) \leq \phi(x)-\phi(T x)
$$

(i.e., $T x \prec_{\phi} x$ ) fixes a, i.e., $T a=a$.

One can now give the graph theory version of the above corollary as follows.

Corollary 2.2 Let $G$ be an acyclic oriented graph with $E(G)$ containing all loops. Suppose that there exists a distance $d$ in $V(G)$ such that $(V(G), d)$ is a metric space and $\phi: V(G) \rightarrow$ $[0, \infty)$ is a map. Consider the partially ordered set $\left(V(G),\left(\prec_{G}\right)_{\phi}\right)$. Assume that a $\in V(G)$ is a minimal element. Then any map $T: V(G) \rightarrow V(G)$ such that for all $x \in V(G)$ with $(x, T x) \in E(G)$

$$
d(x, T x) \leq \phi(x)-\phi(T x)
$$

(i.e., $\left.T x\left(\prec_{G}\right)_{\phi} x\right)$ fixes a, i.e., $T a=a$.

This corollary can be seen as a generalization of Caristi's result endowed with a graph, see [1, Theorem 3]. Indeed, the regular assumptions made in Caristi's theorem imply that 
any chain (for $\prec_{\alpha}$ ) has a lower bound, which is stronger than having a minimal element. Corollary 2.2 in fact contains implicitly a conclusion of the existence of a common fixed point graph. See $[2,15]$ for a similar conclusion in the partial order version.

\section{Main results - Kirk's problem via graphs}

In an attempt to generalize Caristi's fixed point theorem, Kirk [4] has raised the problem of whether a map $T: X \rightarrow X$, such that for all $x \in X$

$$
\eta(d(x, T x)) \leq \phi(x)-\phi(T x)
$$

for some positive function $\eta$, has a fixed point. In fact Kirk's original question was stated when $\eta(t)=T^{p}$ for some $p>1$. In [2], the author gave a good example which answers Kirk's problem in the negative where the order is used implicitly. We present a similar example but in terms of a graph.

Example 3.1 Let $G$ be the graph with a vertex set $V(G)=\left\{x_{n} ; n \geq 1\right\} \subset[0, \infty)$ defined by

$$
x_{n}=1+\frac{1}{2}+\frac{1}{3}+\cdots+\frac{1}{n}
$$

for all $n \geq 1$ and $E(G)=\left\{\left(x_{i}, x_{i}\right),\left(x_{i}, x_{i+1}\right) ; i=1,2,3, \ldots\right\}$. Then $V(G)$ is a closed subset of $[0, \infty)$ and therefore is complete. Let $V(G)$ be endowed with a metric $d: V(G) \times V(G) \rightarrow$ $\mathbb{R}^{+}$defined by the standard distance in $\mathbb{R}$ and $T: V(G) \rightarrow V(G)$ defined by $T x_{n}=x_{n+1}$ for all $n \geq 1$. The graph of $G$ is shown in Figure 1 .

Then

$$
d\left(x_{n}, T x_{n}\right)^{p}=\frac{1}{(n+1)^{p}}=\phi\left(x_{n}\right)-\phi\left(T x_{n}\right),
$$

where $\phi\left(x_{n}\right)=\sum_{i=n+1}^{\infty} \frac{1}{i^{p}}$, for all $n \geq 1$. An easy computation shows that $\phi$ is lower semicontinuous on $[0, \infty)$. Furthermore, one can show that $T$ is nonexpansive, i.e., $d(T x, T y) \leq$ $d(x, y)$ for all $x, y \in V(G)$. And it is obvious that $T$ has no fixed point.

Though the above example gives a negative answer to Kirk's problem, some positive partial answers may also be found. Note that the order approach to Caristi's traditional result is no longer possible. Indeed, if we define on the metric space $V(G)$ the relation $x \prec y$ whenever $\eta(d(x, T x)) \leq \phi(x)-\phi(T x)$, then $\prec$ is reflexive and anti-symmetric. But it is not in general transitive. Of course, if $\eta$ is subadditive, i.e., $\eta(a+b) \leq \eta(a)+\eta(b)$ for any $a, b \in[0, \infty)$, then $\prec$ is transitive. So one may wonder how to approach this general case when $\prec$ is not transitive and therefore $(V(G), \prec)$ is not a partial order set.

Definition 3.1 We say that a map $\eta:[0, \infty) \rightarrow[0, \infty)$ is a well-behaved map if it is nondecreasing, continuous, and such that there exist $c>0$ and $\delta_{0}>0$ such that for any $t \in\left[0, \delta_{0}\right]$ we have $\eta(t) \geq c t$.

Figure $1 G$.

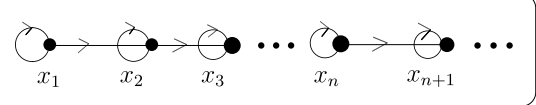


Notice that as $\eta$ is continuous, there exists $\varepsilon_{0}>0$ such that $\eta^{-1}\left(\left[0, \varepsilon_{0}\right]\right) \subset\left[0, \delta_{0}\right]$.

Definition 3.2 Let $G$ be an acyclic oriented graph with $E(G)$ containing all loops. Suppose that there exists a metric $d$ in $V(G)$ such that $(V(G), d)$ is a metric space. One says that $G$ has a minimal vertex (root) $x_{*} \in V(G)$ if $x_{*}$ is a minimal element in $(V(G), \prec)$, i.e., if $x \prec x_{*}$, then we must have $x=x_{*}$, where the relation $\prec$ is defined by

$$
x \prec y \quad \Leftrightarrow \quad \eta(d(x, y)) \leq \phi(y)-\phi(x),
$$

where $\phi: V(G) \rightarrow[0, \infty)$ and $\eta:[0, \infty) \rightarrow[0, \infty)$ are functions.

Theorem 3.1 Let $G$ be an acyclic oriented graph with $E(G)$ containing all loops. Suppose that there exists a metric d in $V(G)$ such that $(V(G), d)$ is a complete metric space. Let $\eta$ be a well-behaved map and $\phi: V(G) \rightarrow[0, \infty)$ be a lower semi-continuous map, then $G$ has $a$ root $x_{*}$.

Proof Set $\phi_{0}=\inf \{\phi(x): x \in V(G)\}$. For any $\varepsilon>0$, set

$$
V(G)_{\varepsilon}=\left\{x \in V(G): \phi(x) \leq \phi_{0}+\varepsilon\right\} .
$$

Since $\phi$ is lower semi-continuous, then $V(G)_{\varepsilon}$ is a closed nonempty subset of $V(G)$. Also note that if $x, y \in V(G)_{\varepsilon}$ and $x \prec y$, then $\eta(d(x, y)) \leq \phi(y)-\phi(x)$, which implies

$$
\phi_{0} \leq \phi(x) \leq \phi(y) \leq \phi_{0}+\varepsilon .
$$

Hence $\eta(d(x, y)) \leq \varepsilon$. Using $c, \varepsilon_{0}$, and $\delta_{0}$ associated with the well-behaved map $\eta$, we get

$$
c d(x, y) \leq \eta(d(x, y)) \leq \phi(y)-\phi(x)
$$

for any $x, y \in V(G)_{\varepsilon_{0}}$ with $x \prec y$. On $V(G)_{\varepsilon_{0}}$ we define the new relation $\prec_{*}$ by

$$
x \prec_{*} y \quad \Leftrightarrow \quad d(x, y) \leq \frac{1}{c} \phi(y)-\frac{1}{c} \phi(x) .
$$

Clearly, $\left(V(G)_{\varepsilon_{0}}, \prec_{*}\right)$ is a partially ordered set with all necessary assumptions to secure the existence of a minimal element $x_{*}$ for $\prec_{*}$. We claim that $x_{*}$ is also a minimal element for the relation $\prec$ in $V(G)$, and hence a root of the graph $G$. Indeed, let $x \in V(G)$ be such that $x \prec x_{*}$. Then we have $\eta\left(d\left(x, x_{*}\right)\right) \leq \phi\left(x_{*}\right)-\phi(x)$. In particular, we have $\phi(x) \leq \phi\left(x_{*}\right)$, which implies $\phi(x) \leq \phi_{0}+\varepsilon_{0}$; i.e., $x \in V(G)_{\varepsilon_{0}}$. As before, we have $\eta\left(d\left(x, x_{*}\right)\right) \leq \varepsilon_{0}$, which implies

$$
c d\left(x, x_{*}\right) \leq \eta\left(d\left(x, x_{*}\right)\right) \leq \phi\left(x_{*}\right)-\phi(x)
$$

which implies $x \prec_{*} x_{*}$. Since $x_{*}$ is a minimal element in $\left(V(G)_{\varepsilon_{0}}, \prec_{*}\right)$, we get $x=x_{*}$. This completes the proof of Theorem 3.1.

Definition 3.3 One says that a mapping $T: V(G) \rightarrow V(G)$ is $G$-edge preserving if

$$
\forall x, y \in V(G) \quad((x, y) \in E(G) \Rightarrow(T x, T y) \in E(G)) .
$$


$T$ is said to be a Caristi-Kirk $G$-mapping if there exists a lower semi-continuous function $\phi: V(G) \rightarrow[0,+\infty)$ and a well-behaved map $\eta$ such that

$$
\eta(d(x, T x)) \leq \phi(x)-\phi(T x), \quad \text { whenever }(T(x), x) \in E(G) .
$$

The next result is a positive partial answer to Kirk's problem via a graph.

Theorem 3.2 Let $G$ be an acyclic oriented graph with $E(G)$ containing all loops. Suppose that there exists a distance $d$ in $V(G)$ such that $(V(G), d)$ is a complete metric space. Let $T: V(G) \rightarrow V(G)$ be a G-edge preserving and a Caristi-Kirk G-mapping. Then $T$ has a fixed point if and only if there exists $x_{0} \in V(G)$ with $\left(T\left(x_{0}\right), x_{0}\right) \in E(G)$.

Proof Define the relation $\prec$ as in Theorem 3.1 on the vertex set $V(G)$. $G$ has all the loops. In particular, if $x_{0}$ is a fixed point of $T$, i.e., $T\left(x_{0}\right)=x_{0}$, then we have $\left(T\left(x_{0}\right), x_{0}\right) \in E(G)$. Assume that there exists $x_{0} \in V(G)$ such that $\left(T\left(x_{0}\right), x_{0}\right) \in E(G)$. Clearly, we have $T\left(x_{0}\right) \prec x_{0}$. Theorem 3.1 implies the existence of a root $x_{*}$ of $G$ and hence we must have $T\left(x_{*}\right)=x_{*}$.

This is an amazing result because the relation $\prec$ is not a partial order. In particular, it is not $\prec_{G}$. Also the minimal point is fixed by any map $T$ that is $G$-edge preserving and a Caristi-Kirk G-mapping. So the fixed point is independent of the map $T$ and only depends on the functions $\phi$ and $\eta$.

\section{Applications of metric spaces with a graph}

The field of mathematics plays a vital role in various fields. One of the important areas in mathematics is graph theory which is used in structural models. These structural arrangements of various objects or technologies lead to new inventions and modifications in the existing environment for enhancement in those fields. This section gives an idea of the implementation of our main results in computer science applications that uses graph theoretical concepts.

\section{A graph model for fault tolerant computing systems}

This section is based on graph theory where it is used to model the fault tolerant system. Here, the computer is represented as $S$ and the algorithm to be executed by $S$ is known as $A$. Both $S$ and $A$ are represented by means of graphs whose vertices represent computing facilities. Algorithm $A$ is executable by $S$ if $A$ is isomorphic to a subgraph of $S$. The author [16] presented a graph model and algorithms for computing systems for fault tolerant systems. These graphs show the computing facility of a particular computation and the interconnection among them. This model is applied directly to the minimum configuration or structure required to achieve fault tolerance to a specified degree. The model is represented in the form of a facility graph. A facility graph is a graph $G$ whose vertices represent system facilities and whose edges represent access links between facilities [16]. A facility here is said to be hardware or software components of any system that can fail independently. Hardware facilities include control units, arithmetic processors, storage units and input/output equipment. Software facilities include compilers, application programs, library routines, operating systems etc. Since each facility can access some other facilities, the real time systems are represented as a facility graph. Figure 2 is a labeled 
Figure 2 Labeled directed facility graph.

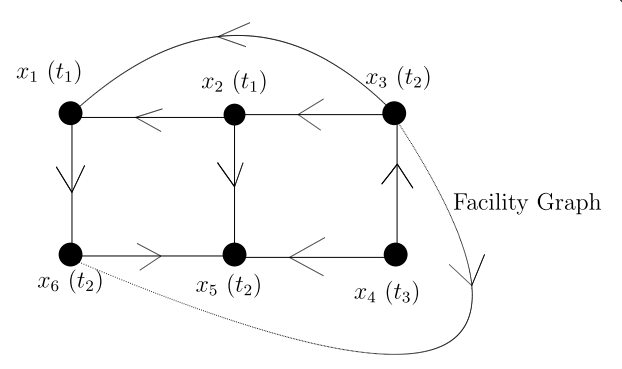

directed facility graph. Facility types are indicated by numbers in parentheses. The graph indicates the types of facilities accessed by other facilities. The vertex $x_{1}$ accesses the vertices $x_{2}$ and $x_{3}$. Similarly, the vertex $x_{5}$ with facility type $t_{2}$ accesses the facility types $t_{1}, t_{3}$ and $t_{2}$ of vertices $x_{2}, x_{4}$ and $x_{6}$, respectively.

\section{Graphical representation of algorithm}

An algorithm will be defined in the form of a facility graph whose vertices represent the facilities required to execute the algorithm and whose edges represent the links required among these facilities. An algorithm $A$ is executable by a computing system $S$ if $A$ is isomorphic to a subgraph of $S$. This means that there is a 1-1 mapping from the vertices of $A$ into the vertices of $S$ that preserves vertex labels and adjacencies between vertices. This implies that $S$ contains all the facilities and connections between facilities required by $A$. So, $A$ can be embedded in $S$.

A $k$-fault $F$ in a system $S$ is the removal of any $k$ vertices $\left\{x_{1}, x_{2}, x_{3}, x_{4}, \ldots, x_{k}\right\}$ from $S$. All edges connected to these vertices are also removed. The resultant graph will be denoted by $S F$. The basic concepts relating to a fault tolerant system is given by the author [16].

(1) A system $S$ is fault tolerant with respect to algorithm $A$ and fault $F$ if $A$ is executable by $S F$.

(2) $S$ is fault tolerant with respect to a set of algorithms $\left\{A_{1}, A_{2}, A_{3}, \ldots, A_{p}\right\}$ and a set of faults $\left\{F_{1}, F_{2}, \ldots, F_{q}\right\}$ if $A_{i}$ is executable by $S F_{j}$ for all $i$ and $j$, where $1 \leq i \leq p$.

If $S$ is $k$-fault tolerant with respect to $A$, then $S$ is $j$-fault tolerant with respect to $A$ for all $j$, where $0 \leq j \leq k$.

Now let $G$ be an acyclic oriented facility graph with $E(G)$ containing all loops. Suppose that there exists a metric $d$ in $V(G)$ such that $(V(G), d)$ is a complete metric space. Let $\eta$ be a well-behaved map and $\phi: V(G) \rightarrow[0, \infty)$ be a lower semi-continuous map, then by Theorem 3.1, $G$ has a root $x_{*}$, i.e., all system faults will be accessed from the root vertex (mother keyboard of the computer) and $S F=\left\{x^{*}\right\}$.

Competing interests

The author declares that he has no competing interests.

\section{Acknowledgements}

The author is grateful to King Fahd University of Petroleum and Minerals for supporting this research. The author would also like to thank Professor MA Khamsi who read carefully the earlier versions of this paper and suggested some improvements.

Received: 17 September 2014 Accepted: 19 November 2014 Published: 08 Dec 2014

References

1. Alfuraidan, MR, Khamsi, MA: Caristi fixed point theorem in metric spaces with a graph. Abstr. Appl. Anal. 2014, Article ID 303484 (2014). doi:10.1155/2014/303484 
2. Khamsi, MA: Remarks on Caristi's fixed point theorem. Nonlinear Anal. 71(1-2), 227-231 (2009)

3. Brondsted, A: Fixed point and partial orders. Proc. Am. Math. Soc. 60, 365-366 (1976)

4. Caristi, J: Fixed point theory and inwardness conditions. In: Applied Nonlinear Analysis, pp. $479-483$ (1979)

5. Halpern, B, Bergman, G: A fixed point theorem for inward and outward maps. Trans. Am. Math. Soc. 130, 353-358 (1968)

6. Browder, FE: On a theorem of Caristi and Kirk. In: Fixed Point Theory and Its Applications (Proc. Sem., Dalhousie University, 1975), pp. 23-27. Academic Press, San Diego (1976)

7. Caristi, J: Fixed point theorems for mappings satisfying inwardness conditions. Trans. Am. Math. Soc. 215, 241-251 (1976)

8. Kirk, WA, Caristi, J: Mapping theorems in metric and Banach spaces. Bull. Acad. Pol. Sci. 23, 891-894 (1975)

9. Ekeland, I: Sur les problèmes variationnels. C. R. Acad. Sci. Paris 275, 1057-1059 (1972)

10. Sullivan, F: A characterization of complete metric spaces. Proc. Am. Math. Soc. 85, 345-346 (1981)

11. Taskovic, MR: On an equivalent of the axiom of choice and its applications. Math. Jpn. 31(6), 979-991 (1986)

12. Chartrand, G, Lesniak, L, Zhang, P: Graphs and Digraphs. CRC Press, New York (2011)

13. Gross, J, Yellen, J: Graph Theory and Its Applications. CRC Press, New York (1999)

14. Brondsted, A: On a lemma of Bishop and Phelps. Pac. J. Math. 55(2), 335-341 (1974)

15. Brondsted, A: Common fixed points and partial orders. Proc. Am. Math. Soc. 77, 365-368 (1979)

16. Hayes, JP: A graph model for fault-tolerant computing systems. IEEE Trans. Comput. 25, 875-884 (1976)

10.1186/1687-1812-2014-240

Cite this article as: Alfuraidan: Remarks on Caristi's fixed point theorem in metric spaces with a graph. Fixed Point Theory and Applications 2014, 2014:240

\section{Submit your manuscript to a SpringerOpen ${ }^{\circ}$ journal and benefit from:}

- Convenient online submission

- Rigorous peer review

- Immediate publication on acceptance

- Open access: articles freely available online

- High visibility within the field

- Retaining the copyright to your article 